\title{
Measuring vibration threshold with a graduated tuning fork in normal aging and in patients with polyneuropathy
}

\begin{abstract}
I S J Martina, R van Koningsveld, P I M Schmitz, F G A van der Meché, P A van Doorn, for the European Inflammatory Neuropathy Cause and Treatment (INCAT) group
\end{abstract}

\begin{abstract}
Objective-To provide clinically useful vibration threshold normal values.

Methods-The graduated Rydel-Seiffer tuning fork was evaluated in 198 healthy controls and 59 patients with a polyneuropathy. The measures were done in triplicate at four locations: the distal interphalangeal joint of the index finger, ulnar styloid process, interphalangeal joint of the hallux, and internal malleolus. The values obtained with this tuning fork in healthy controls and patients with polyneuropathy were compared with the values of an electronic device, the Vibrameter.
\end{abstract}

Results-Vibration sense was better perceived in the arms compared with the legs. There was a significant age related decline of vibration sense at all locations. The values from the Rydel-Seiffer tuning fork and the Vibrameter were significantly correlated in both groups. The sensitivity of these two instruments for the four sites examined in the polyneuropathy group ranged from $29-76 \%$ and $31-73 \%$, respectively and was the highest at the hallux for both instruments.

Department of Neurology, University Hospital Rotterdam, dr Molewaterplein 40 3015 GD, Rotterdam, The Netherlands I S J Martina $R$ van Koningsveld F G A van der Meché P A van Doorn

Department of Statistics, Daniel den Hoed Cancer Centre, University Hospital Rotterdam, Groene Hilledijk 301, 3075 EA, Rotterdam, The Netherlands

P I M Schmitz

Correspondence to: Dr ISJ Martina, Department of Neurology, University Hospital Rotterdam, Dr Molewaterplein 40, 3015 GD Rotterdam, The Netherlands. Telephone 003110 4639222; fax 0031 104633208

Received 2 December 1997 and in revised form

1 April 1998

Accepted 6 April 1998 examination.

(F Neurol Neurosurg Psychiatry 1998;65:743-747)

Keywords: vibration sense; graduated tuning fork; aging

Abnormalities in sensory qualities are frequent complaints of patients with peripheral neuropathies. Impairment of vibration sense, especially starting distally in the limbs, may be a sign of peripheral nerve dysfunction. ${ }^{1}$ Traditionally, a non-graduated tuning fork, which was invented in 1711 and introduced by Bonnafont as a diagnostic test in general medicine, is used for the evaluation of the vibration sense. ${ }^{2}$ This simple instrument measures the presence or absence of vibration, but unfortunately does not quantitatively provide the degree of dysfunction of vibration sense. It is of clinical importance that the vibration sense should be measured quantitatively and consistently. For this purpose several electronic devices have been developed, such as the Biothesiometer, the Optacon, and the computer assisted sensory examination. ${ }^{3-5}$ Although these instruments are useful in experimental studies, they are of little use in daily practice. The size of the apparatus, the duration of examination, the demand on patients' cooperation, and their cost are in sharp contrast with the needs of a simple and valid instrument for use in routine neurological investigation. With the introduction of the $64 \mathrm{~Hz}$ graduated tuning fork by Rydel and Seiffer (Martin, Tuttlingen, Germany) in 1903 it seemed as if all the needs were fulfilled in providing an instrument which was easy to apply, inexpensive, and reliable for quantifying impairment of vibration sense. ${ }^{6}$ However, this tool has been largely neglected by neurologists and thus far, only a few papers have reported on its use..$^{7-14}$ Clinically useful normal values for vibration sense using this tuning fork were provided by one study only. ${ }^{7}$ These values were obtained by examining 73 healthy controls only at the hallux, which hampers their general applicability. Other studies acquired their own normal vibration values, but unfortunately from only a few healthy controls. $^{811}$ No study has provided normal values for the arms. The primary aim of this study was to obtain specific normal vibration threshold values for the Rydel-Seiffer tuning fork at four examination sites in a large number of healthy controls. In addition, the values achieved using the device in healthy controls and in patients with a polyneuropathy were compared with those obtained with an electronic device, the Vibrameter. ${ }^{15}$

\section{Participants and methods}

\section{HEALTHY CONTROLS}

Healthy controls were recruited from hospital personnel, companions (relatives, friends) of patients visiting our outpatient clinic, and from homes for elderly people. Two hundred and fourteen potential controls were interviewed and examined. Those with sensory symptoms, sensory signs including absent vibration sense, a history of alcohol misuse, using drugs which may cause a polyneuropathy or influence their cooperation, and those with a disease which might induce a polyneuropathy, were excluded from the study. The selected participants had a lucid consciousness and their history did not show any mental or psychological illness. Standard neurological examination was performed with special interest in the sensory qualities. Six age groups $(<40,40-49,50-59$, 


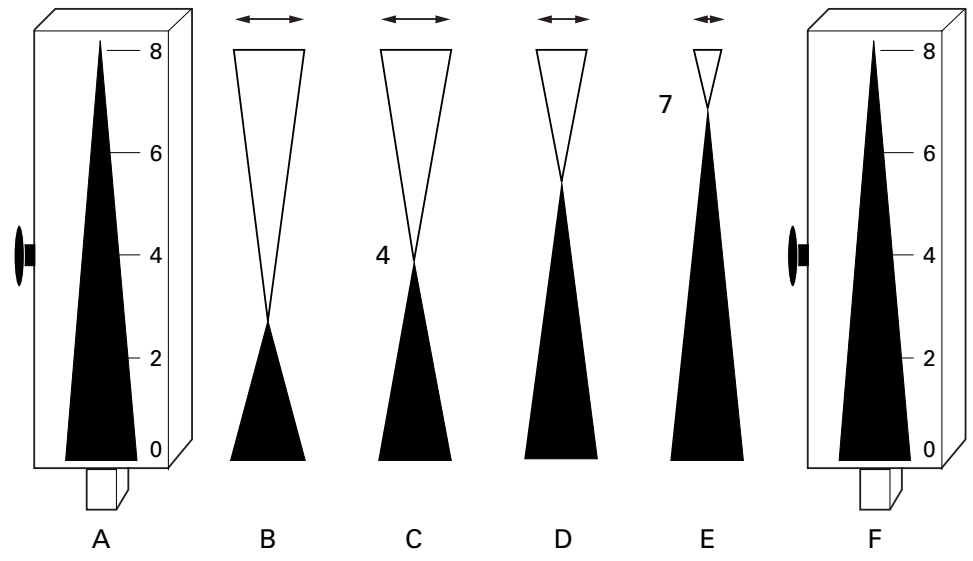

Figure 1 Extremities of the graduated tuning fork at rest $(A)$ and during vibration $(B \rightarrow F)$. Intersection between lower and upper triangles moves from 0 (minimum score) to 8 (maximum score) with decreasing vibration amplitude $(\leftrightarrow)$.

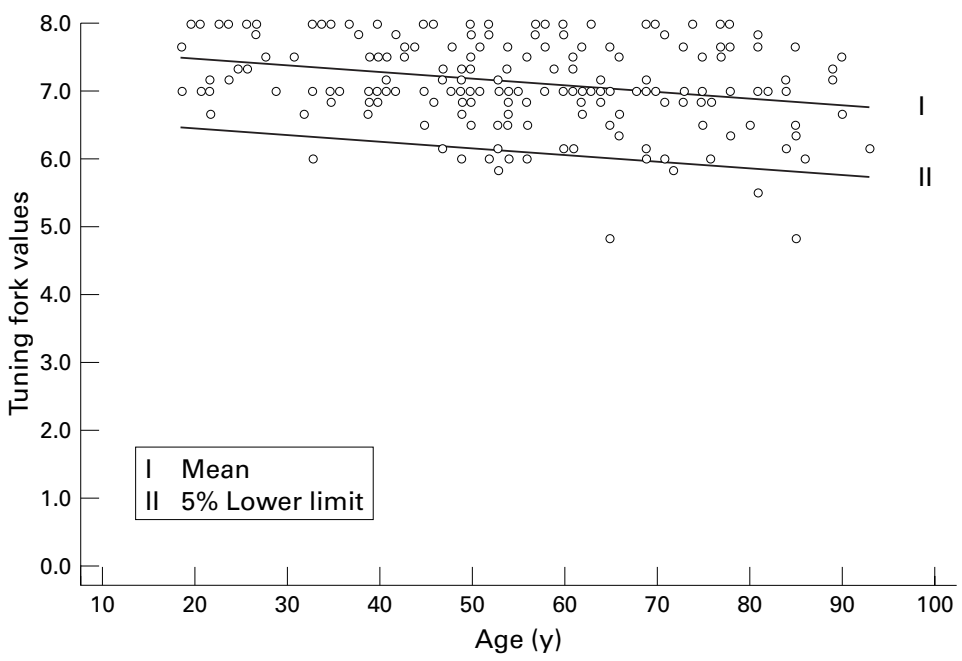

Figure 2 Vibration threshold values obtained by the Rydel-Seiffer tuning fork at the index finger. Residual standard deviation (SDres $=$ residual standard deviation around the mean regression line $)=0.62$. y mean $=7.69-0.01 \times$ age; $y \%=6.67-0.01 \times$ age .

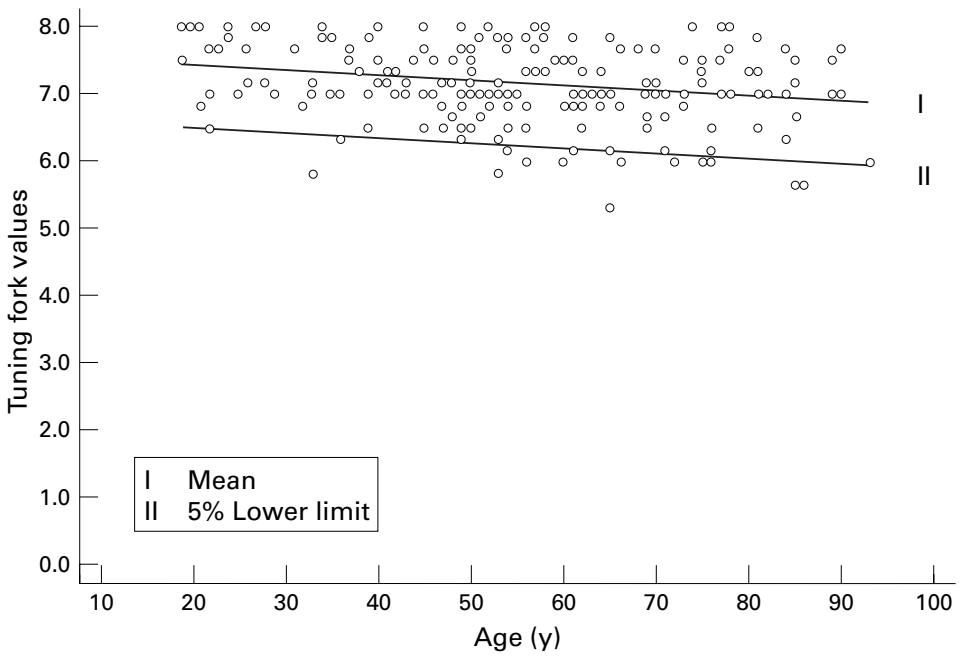

Figure 3 Vibration threshold values obtained by the Rydel-Seiffer tuning fork at the ulnar styloid process. Residual standard deviation (SDres $=$ residual standard deviation around the mean regression line $)=0.55$. y mean $=7.60-0.0079 \times$ age $y 5 \%=6.68-0.0079 \times$ age .

$60-69,70-79, \geqslant 80$ years) were formed. One hundred and ninety eight healthy controls (93 men; 105 women; mean age 55.1 (SD 18.0) years; range 19-93 years) stratified for sex were enrolled in this study. Each age group consisted of about 35 participants. Sixteen subjects (nine men; seven women; mean age 81 (SD 8.5) years; range 65-93 years) were excluded from analysis, based on absent vibration perception in at least one region tested. Eight had an absent vibration sense at the hallux and the internal malleolus, seven only at the hallux, and one only at the internal malleolus. Nine subjects had absent and four decreased ( $-3 /$ $-3)$ ankle jerks. The ankle reflexes were normal in the remaining three patients. These patients were considered to have subclinical disease. The problem of the selection of participants for normal vibration threshold values is discussed later.

\section{PATIENTS WITH POLYNEUROPATHY}

Fifty nine ambulatory patients from our outpatient clinic ( 31 men; 28 women; mean age 56.8 (SD 15.9) years; range 14-87 years) with a clinical and electrophysiologically supported polyneuropathy were enrolled in this study. They were stratified into age group, as described above, and sex. Each age group consisted of about 10 participants. These patients had a mild polyneuropathy with limited sensory disturbances and walking problems. The aetiologies of the polyneuropathies were diabetes mellitus (11 cases), hereditary motor sensory neuropathy type I (five cases), and type II (two cases), systemic disease (three cases), amyloidosis (three cases), drug induced (two cases), thyroid dysfunction (one case) and vitamin $B_{12}$ deficiency (one case). Fourteen patients had a chronic idiopathic axonal polyneuropathy. In 17 cases no cause was determined.

\section{ASSESSMENT TOOLS}

The Rydel-Seiffer tuning fork is a graduated fork which determines the ability of subjects to discriminate various vibration intensities. The two arms of this tuning fork bear calibrated weights at their extremities (fig 1). ${ }^{6}$ A triangle and an arbitrary scale from 0 (minimum score) to 8 (maximum score) imprinted on the weights allow assessment of vibration threshold. Once the arms are swinging, the fork vibrates at $64 \mathrm{~Hz}$ and the triangles on the weights appear double. The intersection of these two virtual triangles moves from 0 to 8 in an exponential way with decreasing vibration amplitude of the arms. The vibration extinction threshold is considered as the nearest value to the apparent point of intersection of the virtual triangles when the subject indicates that vibration is no longer perceived. The Vibrameter (Somedic, Stockholm, Sweden, Type III) is a device which determines vibration sense electronically. It has been extensively described by Goldberg and Lindblom and consists essentially of a hand held vibrating probe which vibrates at $100 \mathrm{~Hz} .{ }^{15}$ The vibration amplitude of this probe increases from zero to a maximum of $399.9 \mu \mathrm{m}$.

TEST PROCEDURES

All participants gave informed consent before the study. All measurements were performed by one investigator $(\mathrm{RvK})$ and the vast majority 


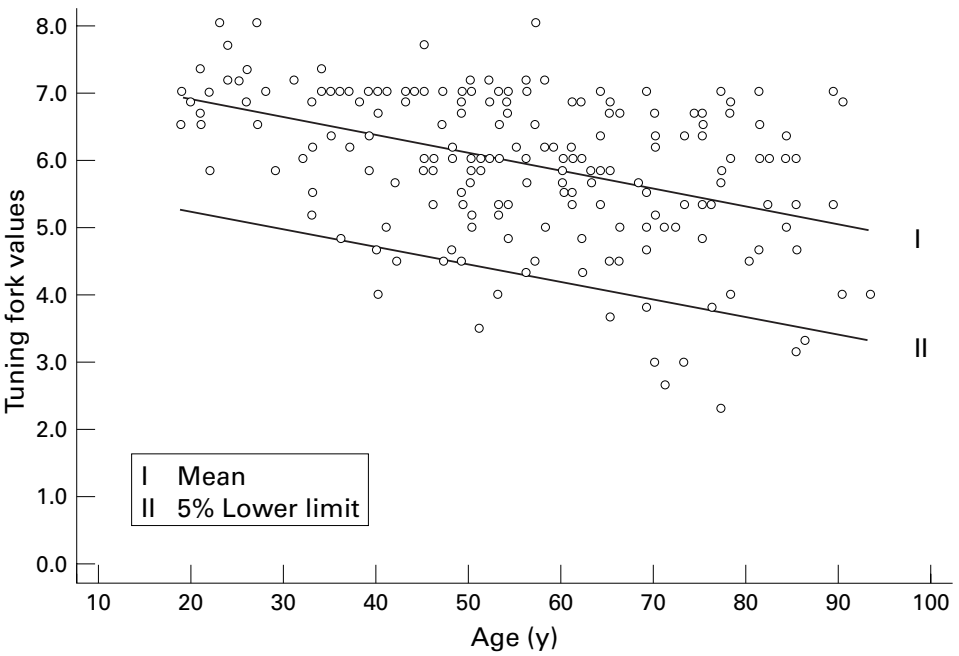

Figure 4 Vibration threshold values obtained by the Rydel-Seiffer tuning fork at the hallux. Residual standard deviation (SDres = residual standard deviation around the mean regression line) $=0.99 . y$ mean $=7.38-0.026 \times$ age; $y \%=5.75-0.026 \times$ age.

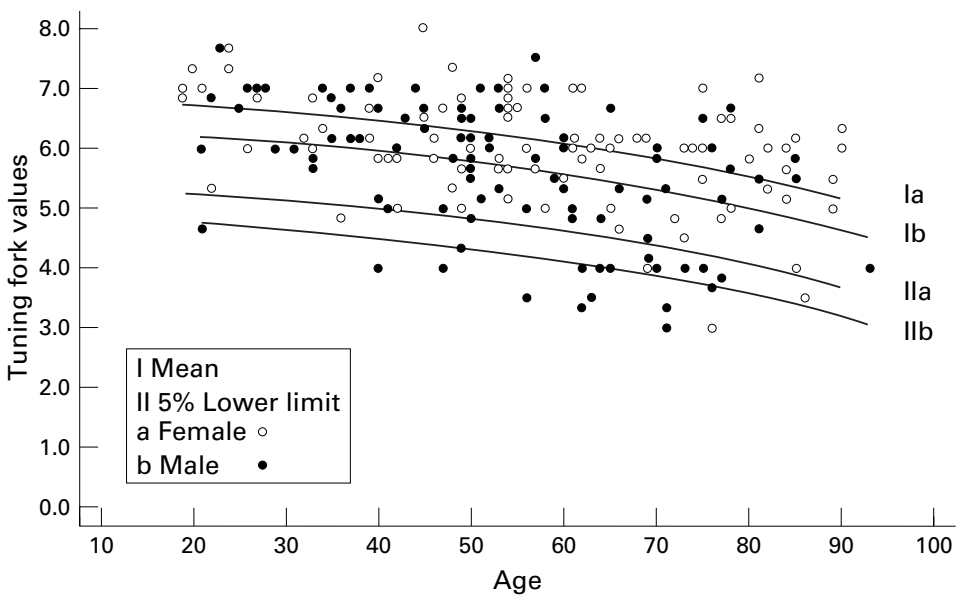

Figure 5 Vibration threshold values obtained by the Rydel-Seiffer tuning fork at the internal malleolus. Residual standard deviation (SDres $=$ residual standard deviation around the mean regression line $)=0.89 ;$ sex $=1$ (male); sex $=0$ (female). $y$ mean $=6.80-0.51 \times$ sex-0.0002 $\times$ age $\times$ age; $y 5$ (female $)=5.33-0.0002 \times$ age $\times$ age; y $5 \%($ male $)=4.82-0.0002 \times$ age $\times$ age.

of the assessments took place in the morning. Vibration threshold was assessed at the dorsum of the distal interphalangeal joint of the index finger, ulnar styloid process, dorsum of the interphalangeal joint of the hallux, and internal malleolus. The examination was always performed at the right side of the body with the exception of those with an injury or malformation at that particular side. The measures were obtained in a quiet, comfortably warm, central heating temperature controlled room at our outpatient clinic or in bedrooms of homes for elderly people $\left(20^{\circ} \mathrm{C}-22^{\circ} \mathrm{C}\right)$. The tuning fork was applied as perpendicular as possible resting on its own weight with the arms of the fork swinging maximally. The participants were asked to lie at ease in a supine position and indicate the moment when they no longer perceived the decreasing vibration stimulus. The readings of three repeated tests were averaged and considered the vibration threshold for that particular site of examination. In addition, vibration sense was assessed with the Vibrameter in half of the selected healthy controls of each age group, stratified for sex ( 48 men; 52 women; total $\mathrm{n}=100$ ), and all patients with polyneuropathy. Vibration threshold was measured according to the method of limits. ${ }^{15}$ The subjects were asked to indicate when the vibration stimulus was felt for the first time (perception threshold) and when this stimulus disappeared again (disappearance threshold). The average of these three paired measurements was considered the vibration threshold at the location investigated. The Vibrameter was applied resting on its own weight and its equilibration was electronically controlled. Each cycle of measurement included catch trials with a resting probe.

STATISTICS

Vibration threshold reference values for the Rydel-Seiffer tuning fork were calculated for the four sites examined, depending on age and sex, using linear and quadratic regression analysis at a chosen specificity of $95 \%$. The $5 \%$ lower limits for the Rydel-Seiffer tuning fork were estimated for each site of examination as the mean $-1.65^{\star}$ Sres. The mean was defined as the mean vibration threshold for a certain age (and sex) and Sres was considered the residual standard deviation around the regression line. The $95^{\text {th }}$ percentile vibration threshold values for the Vibrameter were also calculated. The obtained limits were estimated and further used to determine new specificities and sensitivities for both instruments. A vibration sense was considered to be abnormal if the corresponding value was below the $5 \%$ lower limit when examined with the tuning fork or above the $95^{\text {th }}$ percentile vibration value when investigated with the Vibrameter at the same site of examination. The correlation between the two instruments was analysed by Spearman's rank correlation test. All analyses were performed using Stata 5.0 for Windows 95 (Stata Statistical Software: Release 5.0. 702 University Drive East, College station, TX: Stata Corporation 1997). A p value<0.05 was considered to be significant.

\section{Results}

The whole procedure of assessing vibration sense with the Rydel-Seiffer tuning fork took about 5 minutes, whereas measurements with the Vibrameter needed 10-15 minutes to be completed. In the healthy controls, there was a significant regression between age and the acquired vibration threshold values for the tuning fork at each site of examination $(p<0.0001)$. The corresponding graphics show for each location that the vibration threshold values decreased with aging (figs $2-5$ ). The equations obtained were linear at each site examined, with the exception of the internal malleolus. At this, the vibration thresholds had a quadratic regression on age. Only at this location, a significant regression was also found between the vibration values and sex $(\mathrm{p}<0.0001)$. The calculated vibration thresholds at this side were 0.51 higher (scale $0-8$ ) for women than men. The 5\% lower limit values were calculated for each age (and sex) at each location and additionally translated for use in clinical practice (table 1). The values for vibra- 
Table 1 Normal vibration threshold values (5\% lower limit) in healthy controls (with the Rydel-Seiffer tuning fork)

\begin{tabular}{|c|c|c|c|}
\hline \multicolumn{2}{|c|}{ For the upper extremities } & \multicolumn{2}{|c|}{ For the lower extremities } \\
\hline $\operatorname{Age}(y)$ & Values & Age (y) & Values \\
\hline$\leqslant 40$ & $\geqslant 6.5$ & $\leqslant 40$ & $\geqslant 4.5$ \\
\hline $41-85$ & $\geqslant 6.0$ & $41-60$ & $\geqslant 4.0$ \\
\hline \multirow[t]{2}{*}{$>85$} & $\geqslant 5.5$ & $61-85$ & $\geqslant 3.5$ \\
\hline & & $>85$ & $\geqslant 3.0$ \\
\hline
\end{tabular}

Sites of examination: dorsum of the distal interphalangeal joint of the index finger, the ulnar styloid process, dorsum of the interphalangeal joint of the hallux, and the internal malleolus. Values are $0-8$; the vibration threshold values are presented in rounded numbers.

tion sense were better in the arms than in the legs. In addition, there was a significant $(p<0.0001)$ negative correlation between the results of the two instruments at all sites examined. In the healthy controls, the Spearman rank correlation coefficient (Srcc) ranged from -0.46 at the index finger to a maximum of -0.65 at the hallux. In the group of patients with polyneuropathy, the Srcc showed almost the same pattern, ranging from -0.46 at the internal malleolus to a maximum of -0.71 at the hallux.

When studying the patients with a mild polyneuropathy at a chosen specificity of $95 \%$, the corresponding sensitivity of the RydelSeiffer tuning fork ranged from $29 \%$ at the ulnar styloid process to a maximum of $76 \%$ at the hallux. The Vibrameter had a sensitivity that ranged from $31 \%$ at the index finger to a maximum of $73 \%$ at the hallux (table 2 ). The Rydel-Seiffer tuning fork detected at the locations in the legs a total of eight patients more compared with the Vibrameter. There was no difference in sensitivity between the two devices when examining the patients at the arms. In addition, in participants of 50 years and older the Vibrameter showed a considerable variability between the three obtained vibration values. Variability between the values obtained with the tuning fork was rarely seen.

The vibration values at the four examination sites in the healthy controls and patients with polyneuropathy were combined in each participant to calculate new sensitivities and specificities at a chosen definition for having polyneuropathy. The 5\% lower limit values obtained for the Rydel-Seiffer tuning fork and the $95^{\text {th }}$ percentile vibration values for the Vibrameter were used for this purpose. Describing polyneuropathy as an abnormal vibration sense at at least one of the four sites examined with the Rydel-Seiffer tuning fork resulted in a spe-

Table 2 Sensitivity of the Rydel-Seiffer (RS) tuning fork compared with the Vibrameter in patients with a mild polyneuropathy (chosen specificity of 95\%)

\begin{tabular}{lll}
\hline Location & $\begin{array}{l}\text { RS tuning fork: patients with } \\
\text { abnormal vibration }(n(\%))\end{array}$ & $\begin{array}{l}\text { Vibrameter: patients with } \\
\text { abnormal vibration }(n \\
(\%))\end{array}$ \\
\hline Ulnar styloid process & $17(29)$ & $18(31)$ \\
Index finger & $19(32)$ & $19(32)$ \\
Internal malleolus & $34(58)$ & $28(48)$ \\
Hallux & $45(76)$ & $43(73)$ \\
Sites examined with abnormal sense of vibration: & $13(22)$ \\
0 & $10(17)$ & $10(17)$ \\
1 & $12(20)$ & $18(31)$ \\
3 or 4 & $19(32)$ & $18(31)$ \\
\hline
\end{tabular}

cificity of $80 \%$ and a sensitivity of $83 \%$. The specificity and sensitivity for the Vibrameter were $86 \%$ and $78 \%$, respectively. The corresponding specificity and sensitivity when at least two sites investigated showed an abnormal vibration sense was $94 \%$ and $63 \%$ for the tuning fork, and $98 \%$ and $61 \%$ for the Vibrameter.

\section{Discussion}

The present study provides clinical useful normal values for the vibration sense using the graduated Rydel-Seiffer tuning fork at four sites of examination. These normal values are different from those provided by the literature. Claus et al stated that subjects up to 40 years should score at least $6 / 8$ and those older than 40 at least $4 / 8 .^{7}$ The difference may be explained by the difference in study design: their population consisted of only 73 healthy controls and no information was given on stratification for age and sex. Moreover, examination was performed only at the internal malleolus and thus no values were provided for the arms or the hallux. Three other studies provided normal values for only one site of examination, but unfortunately these values were presented in a graphical way and not translated for general clinical use. ${ }^{8-10}$ Thivolet $e t$ al, however, examined 88 healthy controls at four different sites, but only the hallux was graphically presented. ${ }^{8}$

We found an age related decrease in vibration sense for all four sites of examination. Pearson ${ }^{16}$ was the first to report an age related decrease and subsequently, numerous reports have confirmed this finding. ${ }^{358-1015}$ 17-23 The significance of this decline is not clear and neither is its cause. However, it is known that degenerative transformations of the Pacinian corpuscles, demyelinisation, and fibre loss in peripheral nerves occur with aging. ${ }^{24}$ Degenerative changes also occur in the CNS with advancing age which may also account for the decreasing vibration sense..$^{18}{ }^{24-26}$ The decrease of vibration sense with age is, however, gradual and there is no trend towards absent vibration sense in healthy old persons, not even at the hallux (fig 4). We are inclined to conclude that absence of vibration sense even in elderly people should therefore be considered as abnormal. Many experienced investigators and neurologists make explicit references to a "nonspecific neuropathy of aging" and suggest that these changes occur uniquely during the aging of peripheral nerves. ${ }^{24}{ }^{26}$ Interpretation of an absent distal vibration sense should be carefully taken, and preferably viewed in the context of other symptoms or signs compatible with a polyneuropathy. A thorough evaluation to identify possible causes should always be performed before attributing these abnormalities to aging.

The obtained normal vibration values were higher for the arms than for the legs. These findings have also been reported by others. ${ }^{5} 1718$ A possible explanation for these variances can be found in the differences in length of nerves between the arms and legs. It is known that longer axons are more prone to degeneration of 
distal regions, possibly due to a metabolic abnormality leading to failure of axonal transport and subsequent degeneration. ${ }^{27}$

The sensitivity of the Rydel-Seiffer tuning fork for patients with a polyneuropathy is highly dependent on the selection of patients. In patients with predominantly motor signs and those with only small size myelinated/ unmyelinated nerve disturbances it might be expected that the sensitivity for detecting vibration sense abnormalities using this tuning fork will be low. The vast majority of the patients with a polyneuropathy in the present study had limited sensory disturbances and hardly ambulatory problems. These findings might explain the low sensitivity that was found at the sites examined in the arms and the somewhat higher sensitivity for the two locations investigated in the legs.

The vibration threshold values obtained with the Rydel-Seiffer tuning fork showed a significant correlation with those of the Vibrameter. This correlation was, however, moderate and was probably due to an increasing variability between the values obtained with the Vibrameter in participants 50 years and older. A considerable variability when applying the Vibrameter in elderly people has also been reported earlier. ${ }^{152328}$ A significant correlation between these two instruments has been shown by others. ${ }^{9-11}$ Hotta et al found a good correlation between a variant of the graduated RydelSeiffer tuning fork and the Vibrameter and stated that the tuning fork provided objective and reliable measurements of vibration sense. ${ }^{14}$

The pocket sized Rydel-Seiffer tuning fork detected slightly more abnormalities in the selected group of patients with a polyneuropathy than the Vibrameter. The present study also shows that the Rydel-Seiffer tuning fork is easily applicable, does not require long periods of attention, and measures vibration sense quicker than the Vibrameter.

The clinical relevance of a screening test depends not only on its simplicity and validity, but also on its reliability and sensitivity to register clinically relevant information. Long term follow up of patients with a polyneuropathy will determine the sensitivity of the Rydel-Seiffer tuning fork to changes in time and will indicate whether clinical improvement correlates with improvement in vibration sense. This is currently being evaluated more extensively in a group of patients with immune mediated polyneuropathy with various degrees of severity. Its interobserver and intraobserver reliability and sensitivity to register changes in time are under investigation.

In conclusion, our findings indicate that the Rydel-Seiffer tuning fork is a simple instrument which rapidly measures vibration sense in a semiquantitative way. Clinically useful vibration threshold normal values are provided for this tuning fork. We propose to incorporate this pocket sized instrument in the routine neurological examination.

We thank professor KV Toyka for advising us to use the graduated Rydel-Seiffer tuning fork in the INCAT studies. This study was part of the Biomed-project number BMH4-CT96 0324.

1 Schaumburg HH, Berger AR, Thomas PK. Anatomical classification of peripheral nervous system disorders. In: Disorders of peripheral nerves, $2 n d$ ed. Philadelphia: FA Davis, 1992:10-24

2 Bickerton RC, Barr GS. The origin of the tuning fork. $\mathcal{F} R$ Soc Med 1987;80:771-3.

3 Bloom S, Till S, Sönksen P, et al. Use of a biothesiometer to measure individual vibration thresholds and their variation in 519 non-diabetic subjects. BMF 1984;288:1793-5.

4 Arezzo TC, Schaumburg HH. The use of the Optacon as a screening device. F Occup Med 1980;22:461-4.

5 Dyck PJ, Karnes J, O'Brien PC, et al. Detection thresholds of cutaneous sense in humans. In: Dyck PJ, Thomas PK, Griffin JW, et al, eds. Peripheral neuropathy, 3rd ed. Philadelphia: WB Saunders, 1993:706-28.

6 Rydel A, Seiffer W. Untersuchungen über das vibrationsgefühl oder die sog. "Knochensensibilität" (Pallästhesie). Archiv für

7 Claus D, Carvalho VP, Neundörfer B, et al. Zur unterzuchung des vibrationsempfindens. Nervenarzt 1988;59:138 42

8 Thivolet C, El Farkh J, Petiot A, et al. Measuring vibration sense with graduated tuning fork. Diabetes Care 1990;13: $1077-80$.

9 Liniger C, Albeanu A, Bloise D, et al. The tuning fork revisited. Diab Med 1990; 7:859-64.

10 Crausaz FM, Clavel S, Liniger C, et al. Additional factors associated with plantar ulcers in diabetic neuropathy. Diabet Med 1988;5:771-5.

11 Bergin PS, Bronstein AM, Murray NMF, et al. Body sway and vibration perception thresholds in normal aging and in patients with polyneuropathy. $\mathcal{F}$ Neurol Neurosurg Psychiatry patients with poly

12 Hilz MJ, Claus D, Rösl G, et al. Besserung des temperaturund vibrationsempfindens bei chronischer urämie nach einzeldialyse. Fortschr Neurol Psychiatr 1995;63:264-9.

13 Hilz MJ, Zimmerman P, Rösl G, et al. Vibrameter testing facilitates the diagnosis of uremic and alcoholic polyneuropathy. Acta Neurol Scand 1995;92:486-90.

14 Hotta N, Sugimura K, Tsuchida I, et al. Use of the C64 quantitative tuning fork and the effect of niceritrol in diabetic neuropathy. Clin Ther 1994;16:1007-15.

15 Goldberg JM, Lindblom U. Standardised method of determining vibratory perception thresholds for diagnosing and screening in neurological investigation. $\mathcal{F}$ Neurol Neurosurg Psychiatry 1979;42:793-803.

16 Pearson GHJ. Effect of age on vibratory sensibility. Arch Neurol Psychiatry 1928;20:482-96.

17 De Michele G, Filla A, Coppola N, Bisogno A, et al. Influence of age, gender, height and education on vibration sense. A study by tuning fork in 192 normal subjects. $\mathcal{F}$ Neurol Sci 1991;105:155-8.

18 Wiles PG, Pearce SM, Rice PJS, et al. Vibration perception threshold: Influence of age, height, sex, and smoking, and calculation of accurate centile values. Diabet Med 1991;8: 157-61

19 Merchut MP, Toleikis SC. Aging and quantitative sensory thresholds. Electromyogr Clin Neurophysiol 1990;30:293-7.

20 Critchley M. The neurology of old age. Lancet 1931;i:122130.

21 Kokmen E, Bossemeyer Jr RW, Barney J, et al. Neurological manifestations of aging. $\mathcal{f}$ Gerontol 1977;32:411-9.

22 Steinberg FU, Graber AL. The effect of age and peripheral circulation on the perception of vibration. Arch Phys Med Circulation on the perceptio

23 Thomson FJ, Masson EA, Boulton AJM. The clinical diagnosis of sensory neuropathy in elderly people. Diabet Med 1993;10:843-6.

24 Knox CA. Neuroanatomical changes associated with aging in the peripheral nervous system. In: Albert ML, Knoefel JE, eds. Clinical neurology of aging, 2nd ed. New York: Oxford University Press, 1994:68-78.

25 Howell TH. Senile deterioration of the central nervous system: a clinical study. BMF 1949;1:56-8.

26 Thomas PK. Late life neuropathy: an overview. New Iss Neurosci 1988;1:85-94.

27 Griffin JW, Watson DF. Axonal transport in neurological disease. Ann Neurol 1988;23:3-13.

28 Fagius J, Wahren LK. Variability of sensory threshold determination in clinical use. F Neurol Sci 1981;51:11-27. 